Pathologe 2021 - 42 (Suppl 2):S210-S211 https://doi.org/10.1007/s00292-021-00987-w Angenommen: 3. September 2021 Online publiziert: 5. Oktober 2021 (c) Springer Medizin Verlag $\mathrm{GmbH}$, ein Teil von Springer Nature 2021

\section{Nadja Irina Herbach}

\subsubsection{0-14.09.2020}

Walter Hermanns · Rüdiger Wanke

Institut für Tierpathologie, Ludwig-Maximilians-Universität München, München, Deutschland

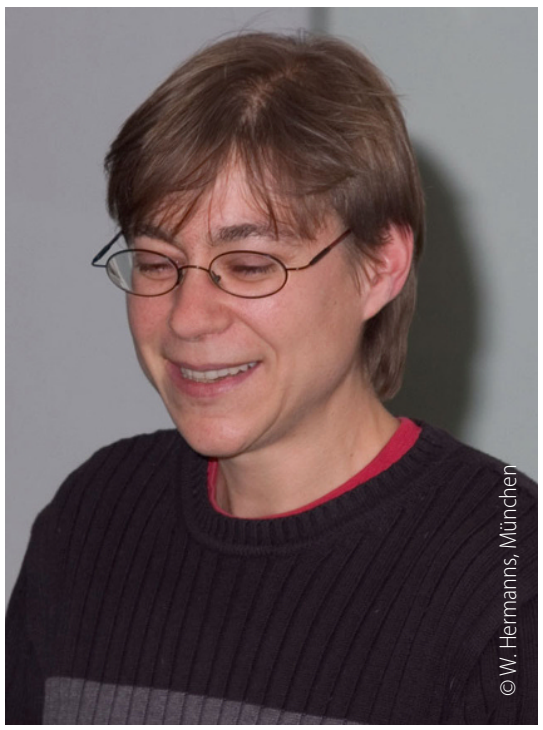

Frau Privatdozentin Doktor med. vet. Nadja Irina Herbach ist kurz nach Vollendung ihres 50. Lebensjahres am. 14. September 2020 nach einer mehrjährigen Tumorkrankheit verstorben.

Frau Herbach wurde am 12. April 1970 in Berlin geboren. Nach Abschluss der Schule absolvierte sie eine zweijährige Ausbildung zur veterinärmedizinisch-technischen Assistentin (VMTA) (1989-1991) in München und arbeitete am Landesuntersuchungsamt für Südbayern in München von 1991 bis 1992 als VMTA. Anschließend absolvierte sie bis 1993 ein Sprachstudium in Irland.

Vom Wintersemester 1993 bis 1999 studierte Frau Herbach Tiermedizin an der Ludwig-Maximilians-Universität München. Bereits als Studentin zeigte sie ein sehr reges Interesse am Fach Pathologie und nahm regelmäßig an den Obduktionsbesprechungen teil. Die Promotion von Frau Herbach erfolgte 2002, ihre Dissertation unter dem Titel „Clinical and pathological characterization of a no- vel transgenic animal model of diabetes mellitus expressing a dominant negative glucose-dependent insulinotropic polypeptide receptor (GIPR dn)" wurde mit summa cum laude bewertet. Ihre Weiterbildung zur Fachtierärztin für Pathologie beendete sie im Februar 2007. Im Jahre 2011 habilitierte sich Frau Herbach an der LMU München. Der Titel ihrer kumulativen Habilitationsschrift lautet „Pathogenesis of diabetes mellitus and diabetic complications". Im Juli 2011 schließlich wurde sie zur Privatdozentin an der LMU München ernannt.

Frau Herbach trat am 1. Mai 1999 in das Institut für Tierpathologie an der LMU München ein, wurde 2002 wissenschaftliche Assistentin und am 1. Mai 2012 zur Akademischen Oberrätin auf Zeit ernannt, eine Stelle, die sie bis zum 30. April 2016 innehatte. Frau Herbach war, neben ihrer mit großer Leidenschaft ausgeübten Tätigkeit in der diagnostischen Pathologie, ganz überwiegend in der Arbeitsgruppe "Molekulare und Experimentelle Pathologie" bei Professor R. Wanke am Institut beschäftigt. Eine langdauernde Krankheit führte dazu, dass Frau Herbach ihre bis dahin sehr erfolgreich und gradlinig verlaufende Karriere nur noch bedingt fortsetzen konnte und so hospitierte sie an mehreren einschlägigen Instituten, unter anderem auch der Humanpathologie.

Den wissenschaftlichen Arbeitsschwerpunkt von Frau Herbach bildete die diabetologische Grundlagenforschung, wobei die Bedeutung von Inkretinhormonen in der Pathogenese des Diabetes mellitus, die Pathomechanismen des Betazellverlustes und die Pathogenese diabetesassoziierter renaler Alterationen ihre Interessenschwerpunkte bildeten. Bereits im Rahmen ihrer Dissertationsarbeit hat sich 
Frau Herbach mit dieser Thematik befasst und danach konsequent zu einer international anerkannten und gefragten Expertin auf dem Gebiet der vergleichenden Diabetesforschung entwickelt. Dies wird durch ihre zahlreichen Publikationen in renommierten Fachjournalen, ihre regelmäßige Gutachtertätigkeit für diabetologische Fachzeitschriften sowie ihre häufigen Einladungen zu Kongressvorträgen eindrucksvoll dokumentiert. Für ihre Studien nutzte sie einerseits mittels transgener Technologie generierte diabetische Mausmodelle und andererseits diabetische Mausmutanten aus dem Münchner ENU-Mausmutageneseprojekt. Die in langjähriger Forschungsarbeit an diesen, mittels "forward genetics" respektive "reverse genetics" erstellten, murinen Diabetesmodellen gesammelten Erfahrungen brachte Frau Herbach auch in Studien an innovativen, für die translationale biomedizinische Forschung generierten porzinen Diabetesmodellen ein, an deren detaillierter Analyse und eingehender Charakterisierung sie maßgeblich beteiligt war. Darüber hinaus war Frau Herbach als Expertin für vergleichende und experimentelle Pathologie in eine Vielzahl interdisziplinärer Kooperationsprojekte mit hochrangigen Forschergruppen im In- und Ausland eingebunden und dabei insbesondere mit nephrologischen, kardiologischen und immunologischen Fragestellungen befasst.

Frau Herbach war von 2009 bis 2011 Mitglied im Exzellenzprogramm LMUMentoring. Im Jahr 2004 wurde ihr der Boehringer-Ingelheim-Award der European Society of Toxicologic Pathology verliehen. Ihre Forschungsergebnisse konnte Frau Herbach dann 2012 im Rahmen der "Aktuellen Habilitationen“ der Deutschen Gesellschaft für Pathologie mit einem Vortrag unter dem Titel „Pathogenese des Diabetes mellitus und diabetischer Spätkomplikationen - Studien an diabetischen Mausmodellen" vorstellen. Mitglied der DGP war Frau Herbach seit dem Jahr 2011.

Mit Frau Privatdozentin Doktor med. vet. Nadja Herbach verliert die Veterinärpathologie und die experimentelle Pathologie eine äußerst profilierte und kenntnisreiche Kollegin, die allen, die sie kannten, stets als kompetente und engagierte Wissenschaftlerin sowie als humorvolle, natur- und tierliebe sowie zutiefst menschliche Persönlichkeit in bester Erinnerung bleiben wird.

The supplement containing this article is not sponsored by industry.

\section{Korrespondenzadresse}

\section{Prof. Dr. Walter Hermanns}

Institut für Tierpathologie, Ludwig-

Maximilians-Universität München

Veterinärstraße 13, 80539 München,

Deutschland

hermanns@patho.vetmed.uni-muenchen.de 